\title{
Rapunzel Syndrome - A Case Report: Trichobezoar as a Cause of Gastric Outlet Obstruction and Intestinal Perforation
}

\author{
Nazia Ishaq Tahir Khaleeq \\ Department of General Surgery, Pakistan Institute of Medical Sciences, Islamabad, Pakistan
}

\section{Keywords}

Gastric outlet obstruction · General surgery · Intestinal perforation $\cdot$ Rapunzel syndrome $\cdot$ Trichobezoar

\begin{abstract}
Rapunzel syndrome is described as a rare manifestation of gastric trichobezoar extending throughout the bowel and is associated with psychiatric disorders. Its incidence in Pakistan is very rare and is found characteristically in adolescent females. Large or complicated trichobezoars are best managed by surgery. Psychiatric follow-up is essential to diminish the frequency of recurrence. We report an unusual case of Rapunzel syndrome causing gastric outlet obstruction and intestinal perforation.

(C) 2021 The Author(s).

Published by S. Karger AG, Basel
\end{abstract}

tinuous ingestion of hair, leading to the impaction of hair together with mucous and food over time. It is associated with many significant behavioral and mental disorders such as trichotillomania, which is a compulsive urge to pull one's hair; anxiety; depression; and bereavement [1].

A trichobezoar that begins in the stomach and extends into the small bowel, sometimes including the right colon, is known as Rapunzel syndrome. This term was coined by Vaughan et al. [2]. The term was derived after the fairy tale, written by the Brothers Grimm, Rapunzel, who has long hair. If gastric outlet obstruction is present, the treatment is surgical, in the form of gastrotomy. In the literature, there are $<40$ cases described, and this is considered to be the most severe form of trichophagia [3]. This case report describes trichobezoar in a young female, causing gastric outlet obstruction and small intestinal perforation, i.e., Rapunzel syndrome.

\section{Case Presentation}

Trichobezoar is defined as collections of hair that has been ingested. As human hair is an indigestible material, it is prone to collect between the mucosal crypts of the stomach due to peristaltic contractions and the smooth surface of the hair [1]. Trichobezoar is formed by con-

karger@karger.com www.karger.com/dmj

Karger $\stackrel{\text { ' }}{5}$

GOPEN ACCESS
(C) 2021 The Author(s)

Published by S. Karger AG, Basel

This is an Open Access article licensed under the Creative Commons Attribution-NonCommercial-4.0 International License (CC BY-NC) (http://www.karger.com/Services/OpenAccessLicense), applicable to the online version of the article only. Usage and distribution for commercial purposes requires written permission.

A 13-year-old female patient presented to our outpatient department with epigastric pain associated with abdominal distention. The abdominal pain lasted for one and a half month and was associated with postprandial emesis. Pain was generalized all over the abdomen, nonradiating in nature and gradually 


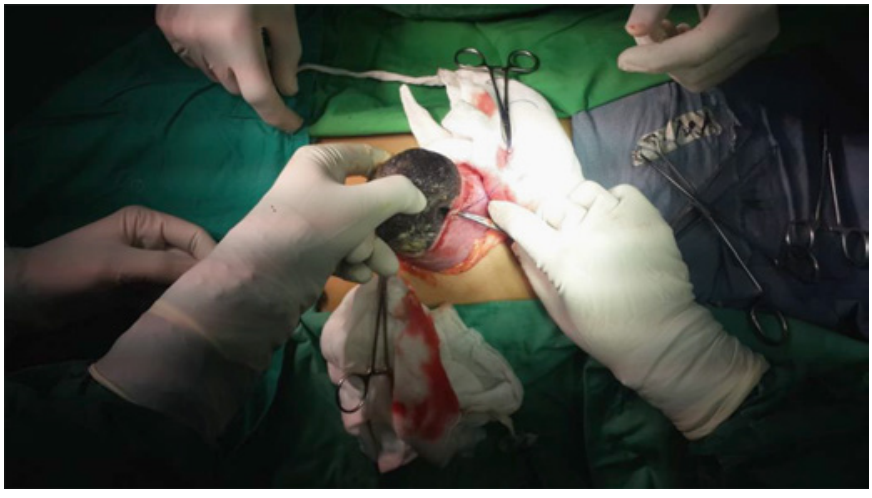

Fig. 1. Longitudinal enterotomy as seen on the anterior wall of the stomach.

worsened in intensity. Vomiting was projectile in nature and contained hair. There was no associated history of constipation or fever. On collateral history, the mother stated that the patient was known to pull her own hair for pleasure (trichotillomania) and then ingesting it (trichotillophagia). The patient also had a compulsive craving for eating nonfood items (pica) such as clay, rocks, and sand.

On general physical examination, the patient was a thin lean young female with mild pallor and no visible signs of malnutrition, and had scanty hair on the lateral aspect of the head. The abdomen was soft and was noncompressible with a large nonmobile, firm, nontender mass in the epigastrium (Lamerton's sign). This extended to the right hypochondrium. It had a smooth surface and rounded borders with no bruit on auscultation. It measured approximately $10 \mathrm{~cm} \times 12 \mathrm{~cm}$. There was no other visceromegaly seen.

Among the complimentary investigations, the patient was anemic with hemoglobin of $11 \mathrm{gm} / \mathrm{dL}$ and corresponding low $\mathrm{MCH}$ and MCV. The patient also had a white cell count of $15.0 \times 10^{9}$, with a CRP of $50 \mathrm{mg} / \mathrm{L}$. An ultrasound of the abdomen showed jejunitis with mesenteric lymph adenitis. A CT scan of the abdomen revealed distended stomach with well-defined area containing filling defect which extended throughout the stomach, duodenum, and jejuna loops. Multiple intussusceptions were seen in small bowel loops in the upper quadrant as well.

In view of her history and examination, she was initially conservatively managed, gastrointestinal decompression was done by passing a nasogastric tube, and adequate hydration was done with intravenous fluids. For the confirmation of diagnosis and possible retrieval, an esophageogastro-endoscopy was performed. A large bezoar was found that was occluding the stomach, and because of the size of the trichobezoar, the removal of the mass via endoscope failed.

For definitive treatment, the patient underwent laparotomy with an upper midline incision to access the abdomen. The stomach was found to be distended with a hard mass inside with a tail extending from the pylorus to the small bowel. A gastrotomy was performed along the anterior wall of the stomach (Fig. 1). A large trichobezoar which had taken up the shape of the stomach was re-

Trichobezoar as a Cause of Gastric Outlet Obstruction and Intestinal Perforation

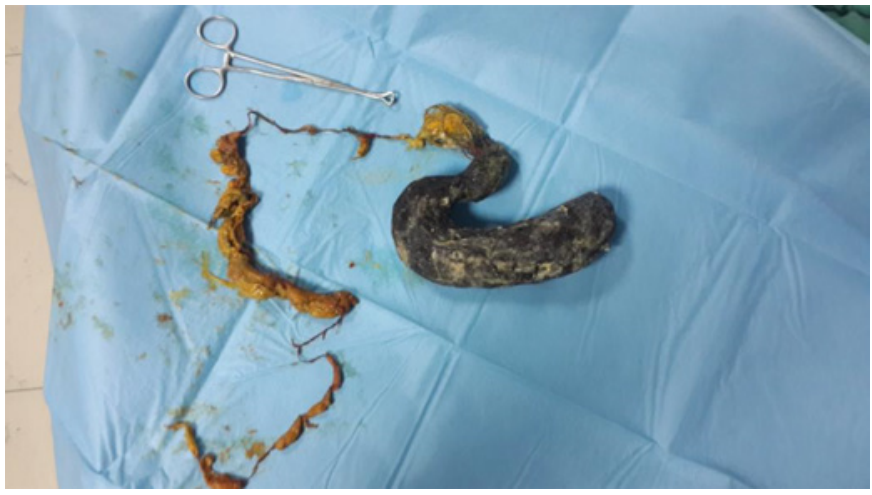

Fig. 2. A large trichobezoar which had taken up the shape of the stomach was retrieved slowly, and the tail was pulled out of the duodenum and jejunum.

trieved slowly, and the tail was pulled out of the duodenum and jejunum (Fig. 2). The rest of the gastrointestinal tract was visualized for daughter fragments, and 2 pinhole-sized perforations were seen on the proximal portion of the jejunum. The perforations had been sealed off by the hair and caused no apparent contamination. Closure of the gastrostomy was done using a stapler, and perforations were closed through primary repair using absorbable sutures. The peritoneal cavity was washed with sterile normal saline and suctioned. The patient did not experience any postoperative complications and recovered completely. On the seventh postoperative day, the patient was successfully discharged home and was referred to the outpatient pediatric psychiatry team.

\section{Discussion}

Rapunzel syndrome is not well documented in the literature. There have been only 45 cases reported, and the latest was published in 2020 by Lyons et al. [4]. The symptoms are vague, and demographics include adolescent females. There should be a high suspicion of trichobezoar if any patient presents with halitosis, patchy alopecia, previous history of trichotillomania, and trichophagia. Complications that can arise are mostly related to malabsorption. These include iron deficiency, protein-losing enteropathy, and megaloblastic anemia [5]. Other common complications include ulceration and perforation. These complications arise mainly due to the lack of blood supply to the stomach and the intestines as the size of the trichobezoar increases.

Many different management plans have been written in the literature for the removal of trichobezoars. They can be removed endoscopically by the use of basket or direct suction, but in cases with a larger size, such as in 
our case report, it is difficult to be removed. Laparoscopic removal has been advised by Nirasawa et al. [6]. However, in view of the high success, low complexity, relatively low complication rate, laparotomy for the treatment of trichobezoars is considered the treatment of choice. This also ensures that the entire gastrointestinal tract can be visualized and evaluated for satellites in a short period of time [7]. To prevent recurrence psychiatry, postoperative management is imperative. This deals with the mental health issues and helps the patient in a hollistic approach.

In our case, we opted for laparotomy due to the size of the trichobezoar and also due to the signs suggestive of multiple intussusceptions. Laparoscopy is known to have less postoperative complications, has better cosmetic results, and reduces hospital duration; however, in this case, we felt that laparotomy gives the advantage of assessing the entire gastrointestinal tract, which is extremely important to help reduce the chances of intestinal obstruction secondary to residual bezoar migration [8].

Analyzing all the data and our experience, we conclude that laparotomy, the gold standard, is most effective in the treatment of patients with Rapunzel syndrome. However, to avoid recurrence, we believe an integrated approach of surgeons, paediatricians, psychiatrists, and gastroenterologists is imperative.

\section{Statement of Ethics}

Study approval statement: This study did not need any local study approval. Consent to publish statement: Written Informed consent was taken from the parents of the patient for publication of this case report and any accompanying images.

\section{Conflict of Interest Statement}

The authors have no conflicts of interest to declare.

\section{Funding Sources}

None.

\section{Author Contributions}

N.I. collected data, and pictures were taken intraoperatively, checking of paper. T.K. contributed to collection of data, analysis, and writing of the paper.

\section{Data Availability Statement}

All data generated in this case report are included in this article. Further inquiries can be directed to the corresponding author.

\section{References}

1 Matejů E, Duchanová S, Kovac P, Moravanský N, Spitz DJ. Fatal case of Rapunzel syndrome in neglected child. Forensic Sci Int. 2009;190(1-3):e5-7.

2 Vaughan ED Jr, Sawyers JL, Scott HW Jr. The Rapunzel syndrome. An unusual complication of intestinal bezoar. Surgery. 1968;63(2): $339-43$.

3 Seker B, Dilek ON, Karaayvaz M. Trichobezoars as a cause of gastrointestinal obstructions: the Rapunzel syndrome. Acta Gastroenterol Belg. 1996;59(2):166-7.
4 Lyons R, Ismaili G, Devine M, Malik H. Rapunzel syndrome causing partial gastric outlet obstruction requiring emergency laparotomy. BMJ Case Rep. 2020;13(1):e232904.

5 Gorter RR, Kneepkens CM, Mattens EC, Aronson DC, Heij HA. Management of trichobezoar: case report and literature review. Pediatr Surg Int. 2010;26(5):457-63.
6 Nirasawa Y, Mori T, Ito Y, Tanaka H, Nobuo S, Atomi Y. Laparoscopic removal of a large gastric trichobezoar. J Pediatr Surg. 1998; 33(4):663-5.

7 Chisholm EM, Leong HT, Chung SC, Li AK. Phytobezoar: an uncommon cause of small bowel obstruction. Ann R Coll Surg Engl. 1992;74(5):342-4.

8 Ahmad Z, Sharma A, Ahmed M, Vatti V. Trichobezoar causing gastric perforation: a case report. Iran J Med Sci. 2016;41(1):67-70. 